\title{
Selection during a selfing programme. II. The effects of two or more rounds of selection
}

\author{
M. A. Cornish
}

School of Biological Sciences, The University of Birmingham, P.O. Box 363, Birmingham B15 2TT, U.K.

Theory presented in a previous paper has been used to provide a description of the responses expected from selection in two or more generations of a selfing series. Optimum two-stages and multi-stage selection programmes are described, for a limited set of circumstances, and compared with the single-stage designs described previously. Simulations show that biases may be expected if epistasis is present or if the number of effective factors is small,. If the number of effective factors is large, then progress from even the most efficient designs will be limited by drift.

\section{INTRODUCTION}

In the previous paper (Cornish, 1990), I considered the response to be expected from a single round of selection during a simplified selfing programme. In that case, it was clear that the predictable improvement in the inbred means was accompanied by a more complex reduction in genetic variation for which an approximate theoretical description was provided. Unlike the case of recurrent selection of an outbreeding population, the loss of genetic variation in a selfing programme is fixed and would therefore reduce the effectiveness of later rounds of selection. The theory developed in the previous paper will be combined here to describe the responses to be expected from twostage and multi-stage selection programmes under a limited set of circumstances. Optimum designs will be determined in these circumstances and compared with the optimum single-stage designs discussed in the first paper. Computer simulations are then used to examine the assumptions implicit in these calculations and to investigate the effects of these designs on a range of quantitative characters.

\section{CALCULATING EXPECTED RESPONSES}

In the simplified selfing programme described in the previous paper, truncation selection was applied to individuals in the $F_{2}$ generation or to family means in later generations. The final response was measured in terms of the mean of a single $\mathrm{F}_{7}$ line. If we again impose a limit of 1000 on the total number of plants between the $F_{2}$ and $F_{6}$ generations inclusive, but now permit selection in two or more generations, then many different designs of programme become possible, depending upon the number of plants allocated to generations and to families.

To illustrate the calculation of the expected total response consider an $F_{2}$ generation of 297 plants from which the best 14 are selected. If these then provide $14 \mathrm{~F}_{3}$ families, each with 50 plants per family, and a single $F_{7}$ line is derived from a randomly chosen member of the best $F_{3}$ family, then 1000 plants are required between the $F_{2}$ and the $F_{6}$. If we consider a quantitative character with a narrow heritability in the $F_{2}$ of 0.5 , with no dominance or epistasis, and with a phenotypic variance in the $\mathrm{F}_{2}$ of 100 , then $D$ (in the notation of Mather and Jinks (1982)) is 100, and $E$ is 50 . From Cornish (1990) the response expected in the $F_{7}$ from selection in the $F_{2}$ is obtained from

$$
R_{1}=i h^{2} \sigma p \text {. }
$$

Selecting 14 from 297 gives an intensity of selection, $i$, of $2 \cdot 0710$ (using Burrows' (1972) approximation), at which value $x=1 \cdot 6545$. Thus:

$$
R_{1}=2 \cdot 0710 \times 0 \cdot 5 \times 10=10 \cdot 3550 .
$$


Following Cornish (1990), the reduction in additive genetic variance caused by selection in the $F_{2}$ is

$$
\begin{aligned}
D_{\mathrm{F}_{2}} & =h^{2} i(i-x) \frac{1}{2} D \\
& =0 \cdot 5(2 \cdot 0710(2 \cdot 0710-1 \cdot 6545)) \times 50 \\
& =21 \cdot 5643 .
\end{aligned}
$$

This is lost from the between families variance in the $F_{3}$ : we assume that the within family variance is unaffected by selection, although the previous paper showed that this may not always be the case. The heritability of the means of $F_{3}$ families of size 50 becomes

$$
\begin{aligned}
h_{F_{3}}^{2} & =\frac{\frac{1}{2} D+(1 / n)\left(\frac{1}{4} D\right)-D_{\mathrm{F}_{2}}}{\frac{1}{2} D+(1 / n)\left(\frac{1}{4} D\right)+(1 / n) E-D_{\mathrm{F}_{2}}} \\
& =\frac{28.9357}{29.9357} \\
& =0.9666 .
\end{aligned}
$$

Selecting 1 family from 14 gives a value of $i$ of 1.7034 (using tables derived from Harter (1961)). The response expected from $F_{3}$ selection becomes

$$
\begin{aligned}
\boldsymbol{R}_{2} & =i h_{\mathrm{F}_{3}}^{2} \sigma_{\mathrm{F}_{3}} \\
& =1.7034 \times 0.9666 \times 5.4717 \\
& =9.0086
\end{aligned}
$$

and the total response expected in $F_{7}$ is therefore

$$
\begin{aligned}
R_{\text {tot }} & =R_{1}+R_{2} \\
& =19 \cdot 3636 .
\end{aligned}
$$

The standardized response is

$$
R=\frac{R_{\mathrm{tot}}}{D^{1 / 2}}=1 \cdot 9364 .
$$

Note that this is 88.0 per cent of the response expected from the optimum $F_{2} / F_{3}$ design in these circumstances (see below). In making these calculations the number of effective factors has been assumed to be infinite, environmental effects are taken to be constant over generations, and environmental effects common to families, genotypeenvironment interactions and biases resulting from non-normality induced by selection have all been assumed to be negligible.

\section{OPTIMUM DESIGNS}

The responses expected from all possible two-stage selection designs under the assumptions described above were calculated in order to determine the optimum design in each case. These designs are presented in table 1 for four levels of narrow heritability. The intensity of selection, $i$, was obtained from tables derived from Harter (loc. cit.) when selection was from populations of 100 or less, and from Burrows' (loc. cit.) approximation when the population was greater than 100 . It can be seen that, for each heritability, the best response involves selection in the $F_{6}$. But the generation in which the first round of selection is best applied ranges from the $F_{2}$ to the $F_{5}$ as the heritability falls. Note also, that in most cases the first round of selection is based on single plants. By practising individual selection in the first round, a high intensity of selection can be achieved whilst still retaining a number of families for the second round of selection. At each heritability, the expected responses are compared with the responses expected from the optimum single-stage designs described in the previous paper (single seed descent to the $F_{6}$ in all cases). For the same total number of plants improvements of up to 17 per cent can be achieved by selecting in two stages rather than in one. Wricke and Weber (1986) found improvements of a similar magnitude in their comparison of one-stage and two-stage selection. The responses presented here are from a limit of 1000 plants between the $F_{2}$ and $F_{6}$ inclusive. If a 1000 plant limit is imposed only on the total of the two selected generations, similar improvements are to be expected over single stage selection of 1000 plants, although in all cases $F_{5} / F_{6}$ selection is best, as is to be expected.

If selection is allowed in any number of generations between the $F_{2}$ and $F_{6}$ then, within the limit of 1000 plants, a very large number of designs are possible. In order to determine the optimum designs at different heritabilities (table 2) an iterative computer approach was necessary. In all but the case with the highest heritability, the maximum response was obtained by applying selection in all five generations. For each design in table $2, m$ is the optimum number of families, and $n$ is the optimum family size. At a heritability of $0 \cdot 25$, for example, it is necessary to select 156 out of $552 \mathrm{~F}_{2}$ individuals, derive a single $\mathrm{F}_{3}$ individual from each of these, select the best $52 \mathrm{~F}_{3}$ individuals and so on, until a single $\mathrm{F}_{7}$ line is derived from a randomly chosen member of the best $F_{6}$ family. As is to be expected, as narrow heritability declines, the value of selection in the early generations falls, and the emphasis shifts towards selecting between well replicated families in later generations. As in the case of two-stage selection, the optimum family size in the early generations remains low, even 


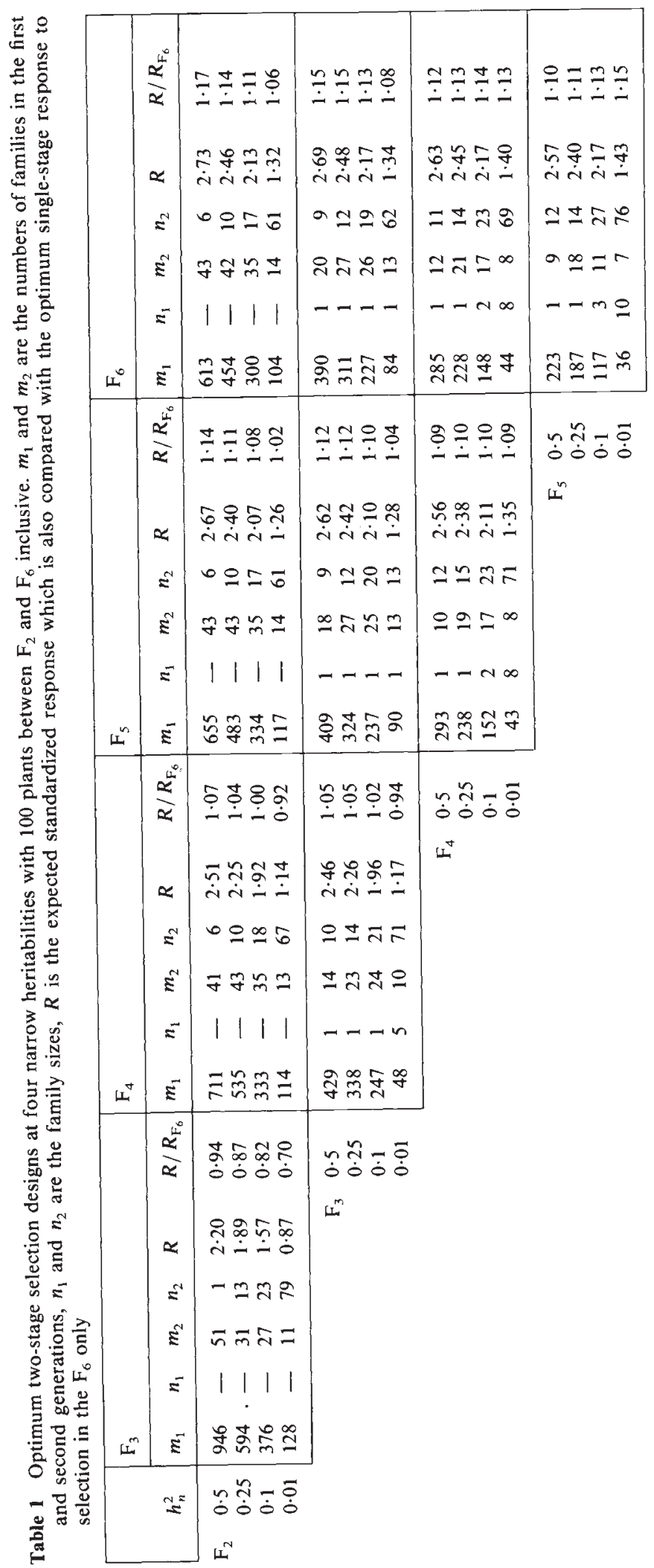


Table 2 Optimum multi-stage selection designs at four different heritabilities and 1000 plants between $\mathrm{F}_{2}$ and $\mathrm{F}_{6}$ inclusive. $m$ is the optimum number of families and $n$ the family size in each generation. $R$, the expected standardized selection response, is compared with the responses from $F_{6}$ selection and from the optimum two-stage selection designs

\begin{tabular}{|c|c|c|c|c|c|c|c|c|c|}
\hline & \multicolumn{9}{|c|}{ Narrow heritability } \\
\hline & \multicolumn{2}{|l|}{0.5} & \multicolumn{2}{|c|}{$0 \cdot 25$} & \multicolumn{2}{|l|}{$0 \cdot 1$} & \multicolumn{2}{|c|}{0.01} & \\
\hline & $m$ & $n$ & $m$ & $n$ & $m$ & $n$ & $m$ & $n$ & \\
\hline $\mathrm{F}_{2}$ & 726 & 一 & 552 & - & 380 & - & 134 & - & \multirow{6}{*}{$\begin{array}{l}1000 \\
\text { plants }\end{array}$} \\
\hline $\mathrm{F}_{3}$ & 110 & 1 & 156 & 1 & 152 & 1 & 71 & 1 & \\
\hline $\mathrm{F}_{4}$ & 23 & 1 & 52 & 1 & 66 & 1 & 40 & 3 & \\
\hline $\mathrm{F}_{5}$ & 23 & 3 & 22 & 5 & 32 & 6 & 18 & 15 & \\
\hline $\mathrm{F}_{6}$ & 4 & 18 & 5 & 26 & 6 & 35 & 5 & 81 & \\
\hline $\mathrm{F}_{7}$ & 1 & $\infty$ & 1 & $\infty$ & 1 & $\infty$ & 1 & $\infty$ & \\
\hline$R$ & \multicolumn{2}{|c|}{$2 \cdot 82$} & \multicolumn{2}{|c|}{$2 \cdot 64$} & \multicolumn{2}{|c|}{$2 \cdot 38$} & \multicolumn{2}{|c|}{1.53} & \\
\hline$R / R_{\mathrm{F}_{6}}$ & \multicolumn{2}{|c|}{$1 \cdot 20$} & \multicolumn{2}{|c|}{$1 \cdot 22$} & \multicolumn{2}{|c|}{$1 \cdot 25$} & \multicolumn{2}{|c|}{$1 \cdot 24$} & \\
\hline$R / R_{2 \text {-stage }}$ & \multicolumn{2}{|c|}{$1 \cdot 03$} & \multicolumn{2}{|c|}{$1 \cdot 07$} & \multicolumn{2}{|c|}{$1 \cdot 10$} & \multicolumn{2}{|c|}{1.07} & \\
\hline
\end{tabular}

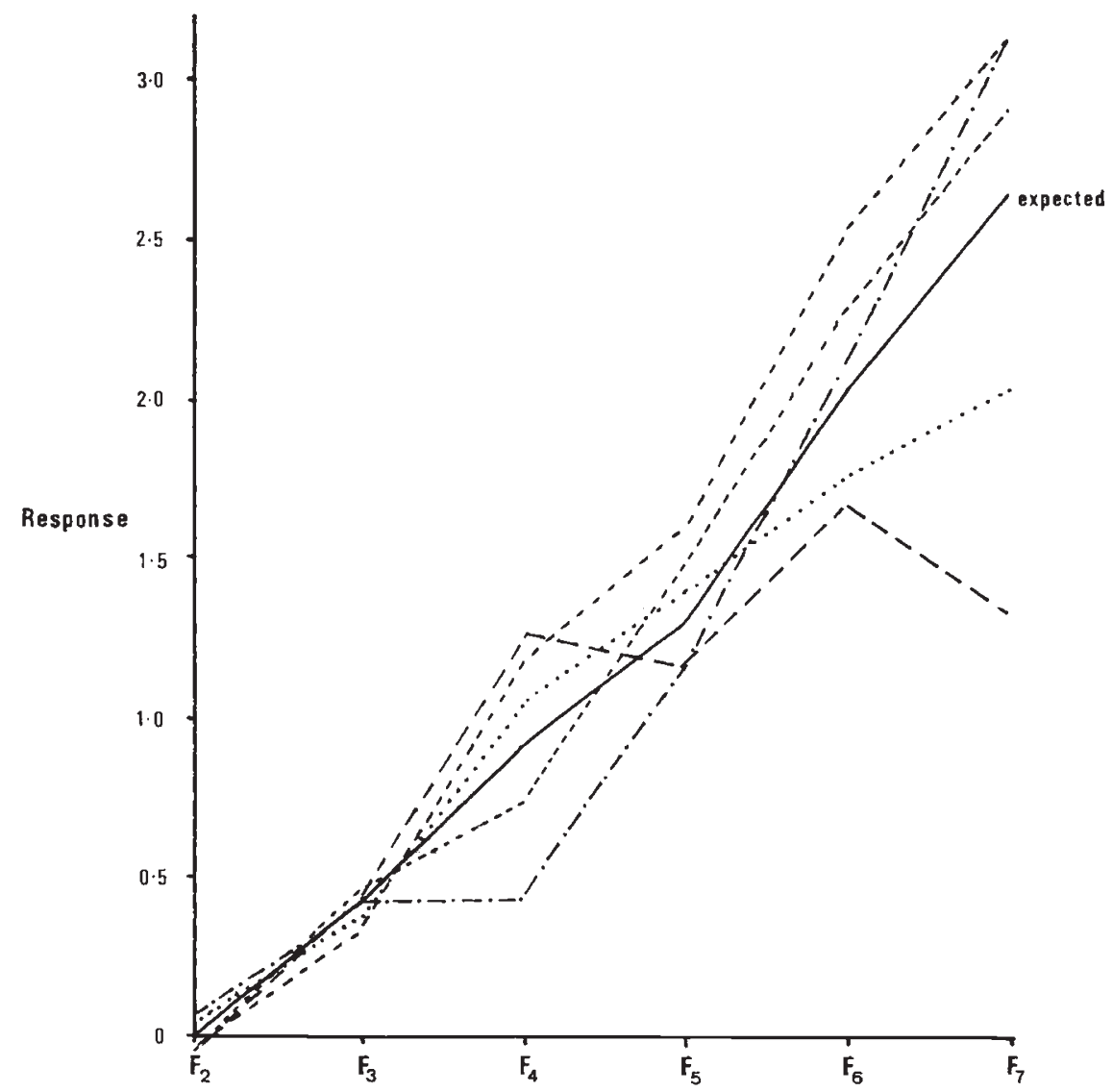

Figure 1 Cumulative responses from five repeat simulations of the optimum multi-stage selection design at a narrow heritability of 0.25 (table 2). Twenty independent genes of equal effect were simulated without dominance or epistasis. 
when the narrow heritability is low. Also in table 2 the responses expected from these multi-stage programmes are compared with those expected from the optimum two-stage designs described above, and the optimum single-stage designs described previously. Within the limitations imposed in this study, we would expect responses from these multi-stage programmes to be between 20 and 25 per cent better than those expected from the best single stage designs, even though the same total number of plants has been used. It can be seen that the advantages of multi-stage designs over two-stage designs are not great: a large proportion of the total response expected by selecting in every generation can be obtained from two well designed rounds of selection.

\section{SIMULATION RESULTS}

The optimum multi-stage selection programmes were simulated using the computer program described in the previous paper. Fig. 1 shows the

Table 3 Results of 200 simulations of the optimum multi-stage selection designs ( 1000 plants maximum between the $F_{2}$ and $F_{6}$ ). The mean and variance of the observed standardized responses are given along with the average number of genes fixed for the increasing allele, and the average number of heterozygous genes. The six characters are described in the text

\begin{tabular}{|c|c|c|c|c|c|}
\hline & $\begin{array}{l}h_{n}^{2} \\
\text { expected } \\
\text { mean }\end{array}$ & $\begin{array}{l}0.5 \\
2.82\end{array}$ & $\begin{array}{l}0.25 \\
2.64\end{array}$ & $\begin{array}{l}0.1 \\
2.38\end{array}$ & $\begin{array}{l}0.01 \\
1.53\end{array}$ \\
\hline \multirow[t]{4}{*}{$k=20$} & mean & 2.75 & $2 \cdot 65$ & $2 \cdot 41$ & 1.53 \\
\hline & variance & 0.18 & 0.24 & 0.26 & 0.54 \\
\hline & $A A$ & 16.01 & $15 \cdot 67$ & $15 \cdot 24$ & $13 \cdot 29$ \\
\hline & $A a$ & 0.29 & 0.26 & 0.30 & 0.26 \\
\hline \multirow[t]{4}{*}{$k=10$} & mean & $2 \cdot 70$ & $2 \cdot 52$ & $2 \cdot 29$ & $1 \cdot 51$ \\
\hline & variance & 0.14 & 0.18 & 0.25 & 0.53 \\
\hline & $\boldsymbol{A A}$ & 9.22 & 8.93 & $8 \cdot 55$ & $7 \cdot 32$ \\
\hline & $\mathrm{Aa}$ & 0.11 & 0.12 & 0.17 & 0.12 \\
\hline \multirow[t]{4}{*}{$k=5$} & mean & $2 \cdot 23$ & $2 \cdot 21$ & $2 \cdot 10$ & 1.46 \\
\hline & variance & 0.04 & 0.02 & 0.10 & 0.46 \\
\hline & $A A$ & 4.99 & 4.97 & $4 \cdot 83$ & $4 \cdot 11$ \\
\hline & $A a$ & 0.00 & 0.01 & 0.03 & 0.04 \\
\hline \multirow{4}{*}{$h=d$} & mean & 2.83 & 2.64 & $2 \cdot 37$ & 1.58 \\
\hline & variance & 0.23 & 0.26 & 0.35 & 0.55 \\
\hline & $A A$ & 15.91 & $15 \cdot 54$ & $14 \cdot 85$ & $13 \cdot 10$ \\
\hline & $A a$ & 0.42 & 0.37 & 0.45 & 0.45 \\
\hline \multirow[t]{4}{*}{ i positive } & mean & $3 \cdot 25$ & $3 \cdot 16$ & 2.93 & $1 \cdot 78$ \\
\hline & variance & 0.37 & 0.41 & 0.50 & 0.79 \\
\hline & $A A$ & 16.05 & 15.96 & $15 \cdot 49$ & $13 \cdot 46$ \\
\hline & $A a$ & 0.24 & 0.24 & 0.31 & 0.34 \\
\hline \multirow{4}{*}{$i$ negative } & mean & $2 \cdot 20$ & 2.06 & 1.88 & $1 \cdot 33$ \\
\hline & variance & 0.09 & 0.12 & 0.15 & 0.34 \\
\hline & $A A$ & $15 \cdot 80$ & $15 \cdot 46$ & $14 \cdot 72$ & 13.09 \\
\hline & $A a$ & 0.26 & 0.27 & 0.27 & 0.34 \\
\hline
\end{tabular}

results of five repeat runs of a simulation of the optimum design at an initial heritability of 0.25 , described in table 2. Twenty independent genes of equal effect were simulated in the presence of normally distributed environmental variation, but in the absence of dominance and epistasis. The solid line shows the expected, cumulative, standardized response. The five simulations followed the expected line approximately, although sampling error led to a range of final responses. The distribution of the final responses from 200 repeat simulations of this design is shown in fig. 2, where it can be compared with 200 simulations of the optimum single-stage design ( $F_{6}$ selection) for the same characters described previously (Cornish, 1990). The average improvement of the multi-stage design over single seed descent can be seen, although the distributions show considerable overlap. The multi-stage design gives a slightly less variable response.

The results of 200 repeat simulations of the four optimum multi-stage designs, applied to a range of quantitative characters, are presented in table 3. In addition to the observed means and variances of each set of simulations, the average number of genes fixed for the increasing allele, and the average number of genes remaining heterozygous are also given. With a character controlled by 20 genes, without dominance or epistasis, the results agreed closely with the expected responses; even at the highest heritability only 16 of the 20 genes were, on average, fixed for the desired allele, whilst the frequency of heterozygous loci was slightly lower than would be expected in an $F_{7}$ line derived without selection $(0.3125)$. With only 10 genes the final response was reduced slightly, and a further reduction was seen when only five genes were simulated.

The addition of complete dominance $(h=d)$ to the 20 gene character made little difference to the average final response, although the frequency of heterozygous loci rose slightly. It follows that the effects of partial dominance and ambirectional dominance would have been even less important. Complete dominance was reported previously (Cornish, 1990) as having only a small effect on simulated single-stage selection. On the other hand, as was also reported previously, epistasis between homozygous genes distributed equally over all 190 gene pairs $(I / I+D=0.01)$ generated sufficient skewness to produce large departures from expected; the final response and its variance increased when $i$ was positive, but decreased when $i$ was negative. The cumulative responses of these simulations at a heritability of 0.25 are shown in 


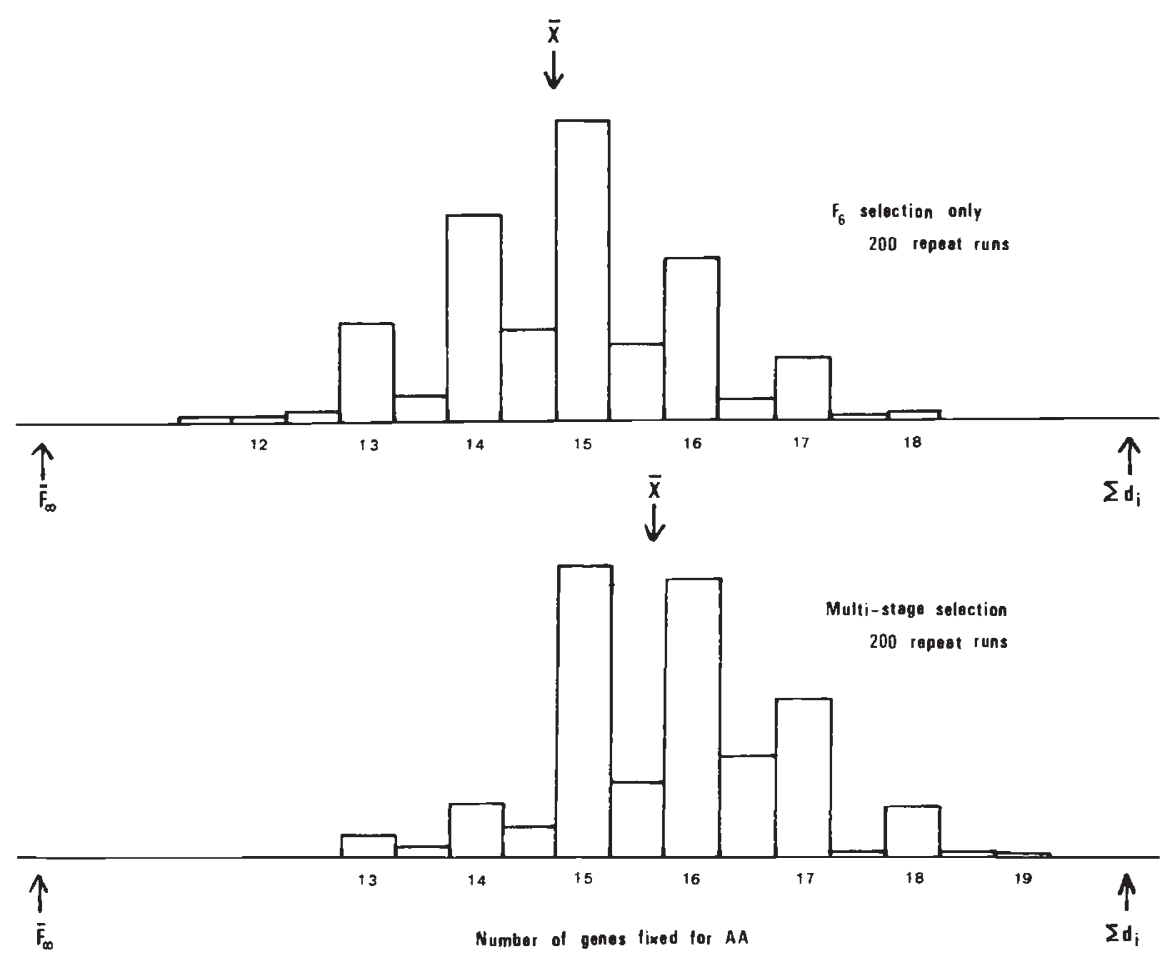

Figure 2 Distributions of the single $F_{7}$ lines obtained from 200 repeat runs of the optimum $F_{6}$ selection and multi-stage selection designs at a narrow heritability of 0.25 . The character is the same as in fig. 1 . The expected mean of the inbreds derived without selection and of the best possible inbred line are shown.

figs 3 and 4. Only considerable skewness caused by epistasis, or a low number of genes, led to serious departures from expected, although it must be noted that even these biases were smaller than the sampling errors revealed in figs 1 and 2 .

\section{DISCUSSION}

By considering the effects of selection on both means and variances, and by taking a very simplified set of circumstances, it has been possible to predict the responses obtained from simulated multi-stage selection programmes. Epistasis and a small number of genes led to marked departures from expected. Extreme forms of dominance were found to be unimportant, and it follows, therefore, that less extreme forms such partial dominance and ambidirectional dominance are also unimportant in these circumstances. The approach used assumed that the genotypes generated by selection remained normally distributed, and that the genetic variance generated by segregation after selection was unaffected. These assumptions appear to have been justified.

Theoretical arguments have been used to determine optimum programme designs in defined situations. The designs presented illustrate the balance that must be achieved from an efficient multi-stage selection programme. If an early round of selection is too intense, then later efforts may be wasted as a result of the genetic variation lost in the early stages. Alternatively, if little or no selection is applied in the early stages in order to preserve genetic variation, then opportunities for improvement may have been missed. By permitting selection in any generation between the $F_{2}$ and the $F_{6}$ inclusive, responses were obtained that were between 20 and 25 per cent better than those from $\mathrm{F}_{6}$ selection alone. In making these comparisons, a total limit of 1000 plants was imposed, and plants counted equally whether or not selection was applied to them. This would, of course, not be the case in practice.

Although only the best design of a selection programme was presented for each heritability 


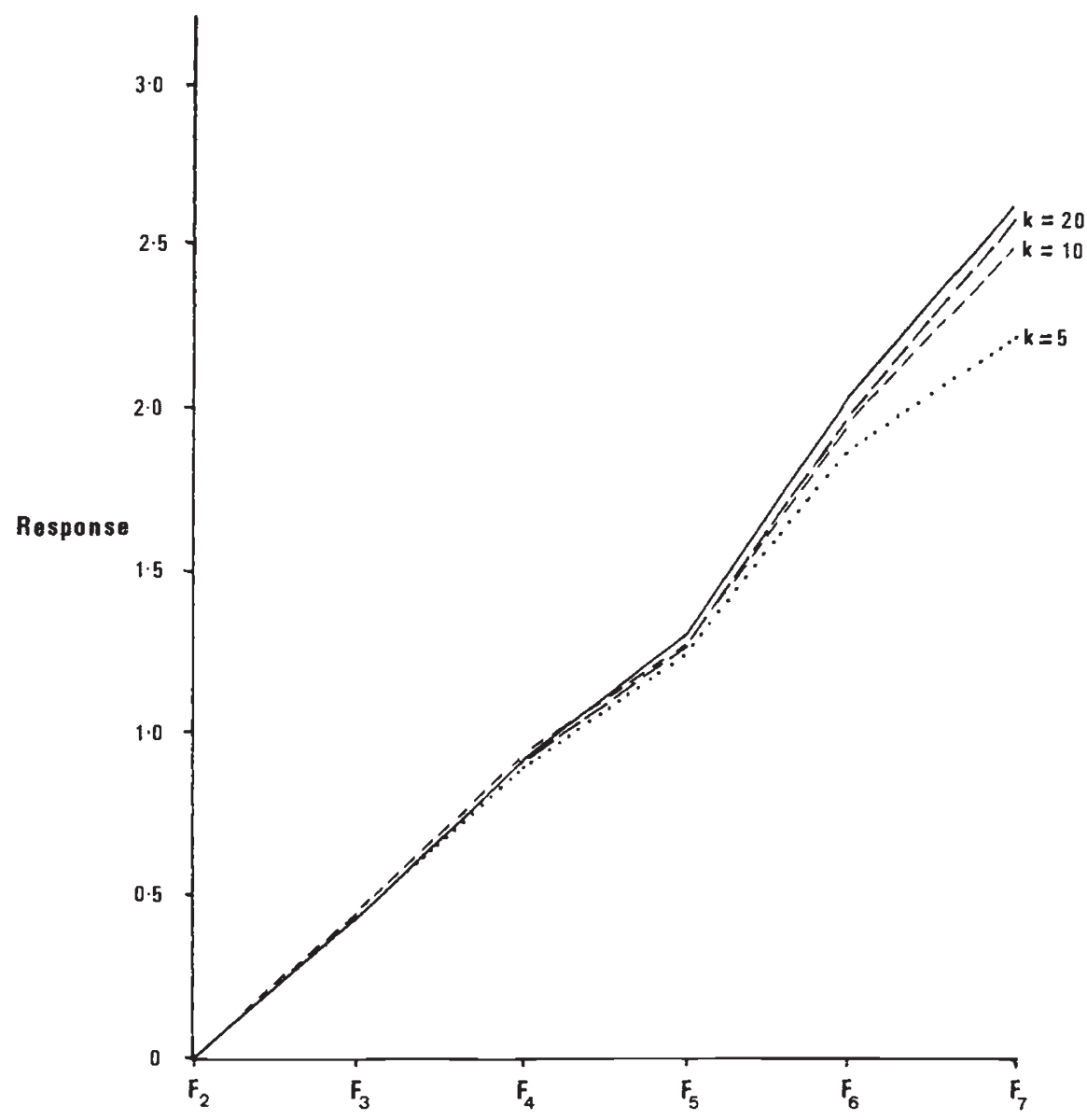

Figure 3 Mean cumulative responses of 200 repeat simulations of the optimum multi-stage design with a narrow heritability of $0 \cdot 25$, and with 5,10 , and 20 independent genes, compared with the expected response.

studied, there were, in each case, a very large number of similar designs giving responses very close to the optimum. This is because, as Wricke and Weber (1986) also found, the curve around the optimum is always flat: a design which is optimal at a heritability of 0.25 is still likely to be very efficient, although suboptimal, at a heritability of $0 \cdot 1$. Hence, accurate estimates of heritabilities would not appear to be essential when designing efficient selection programmes.

It can be seen from the computer simulations that progress was limited even from the most efficient designs. When the number of effective factors was large the probability of obtaining the very best inbred, or even an individual approaching the best, from a single cycle of inbreeding was very small. This is a manifestation of the problem of genetic drift that has been discussed previously in this context (Snape and Simpson, 1984; Sneep, 1977). By maximising the final response, the optimum designs presented here minimized drift, but when the number of effective factors was large drift limited progress with all programmes.

The implications that can be drawn from the details of these multi-stage selection designs are limited by the simplifications adopted. They do, however, serve to illustrate an approach to designing efficient selfing programmes that results from an understanding of responses to selection in terms of both means and variances. If evidence can be obtained from a range of species to support these theoretical arguments, this approach could, in principle, be developed for application to actual breeding programmes. 


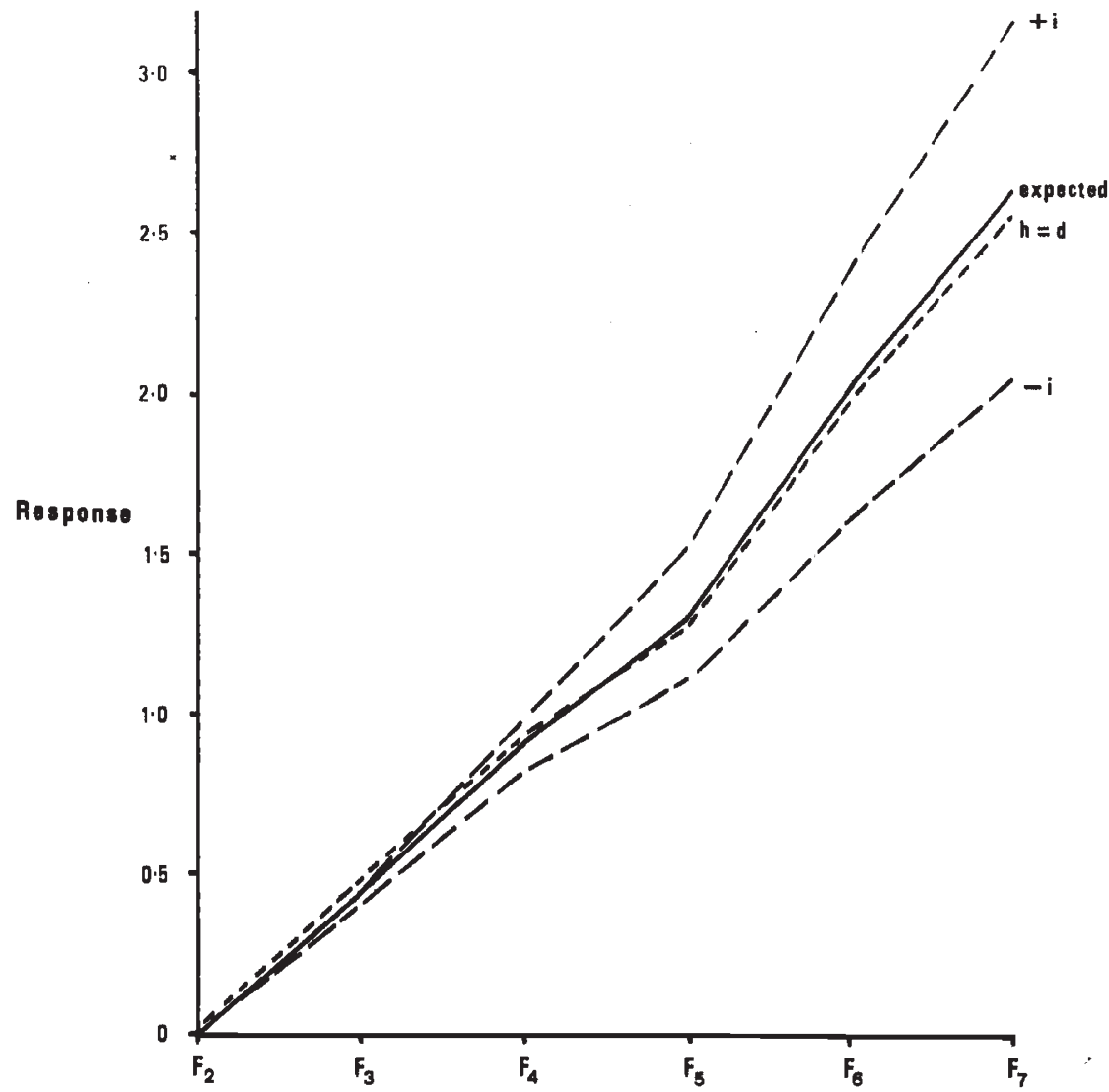

Figure 4 Mean cumulative response of 200 simulations with complete dominance ( $h=d$ at all 20 genes), and with positive and negative $i$ type epistasis $(I /(D+I)=0.01)$ compared with the expected response.

Acknowledgements I am indebted to Dr M. J. Lawrence for his valuable advice during the preparation of this paper.

\section{REFERENCES}

BURROWS, P. M. 1972. Expected selection differentials for directional selection. Biometrics, 28, 1091-1100.

CORNISH, M. A. 1990. Selection during a selfing programme. I. The effects of a single round of selection. Heredity, 65 , 201-211.
HARTER, H. L. 1961. Expected values of normal order statistics. Biometrics, 48, 151-165.

MATHER, K. AND JINKS, J, L. 1982. Biometrical Genetics, 3rd edn. Chapman and Hall, London.

SNAPE, J. W. AND SIMPSON, E. Early generation selection and rapid generation advancement methods in autogamous crops. Proceedings of 10 th congress, EUCARPIA, Pudoc, Wageningen, 82-86.

SNEEP, J. 1977. Selection for yield in early generations of self-fertilizing crops. Euphytica, 26, 27-30.

WRICKE, G. AND WEBER, W. E. 1986. Quantitative Genetics and Selection in Plant Breeding. Gruyter, Berlin. 